\title{
The Dynamic Bipolar Electrode in Polymer Light-Emitting
}

\section{Electrochemical Cells}

Shiyu Hu and Jun Gao*

Department of Physics, Engineering Physics and Astronomy, Queen's University, Kingston, Ontario, K7L 3N6, Canada

ABSTRACT: The redox doping reactions in polymer light-emitting electrochemical cells (PLECs) have been analyzed to gain insight into a new type of bipolar electrode (BPE) formed within the cells. Planar (lateral) PLECs are constructed on a glass substrate with an exposed active polymer layer contacted by two aluminum (Al) driving electrodes at $11 \mathrm{~mm}$ apart. A horizontal array of 13 silver ( $\mathrm{Ag}$ ) microdiscs is deposited in-between the driving electrodes. When a $200 \mathrm{~V}$ bias is applied between the driving electrodes, the electrically floating Ag discs functioned as BPEs to induce redox $\mathrm{p}$ - and n-doping reactions in polymer layer that initially occur at the extremities of the BPEs. The doping reactions are visualized as strong photoluminescence quenching of the luminescent polymer. Over time, the doped regions grow in size, and the silver disc BPEs transform into dynamic, composite BPEs consisting of a p- and ndoped polymer conductor connected by the initial BPE disc. Unlike a homogenous BPE, the dynamic BPE is not a quasi-equipotential body under operation. Moreover, the $\mathrm{n} / \mathrm{p}$ area ratios of all 13 BPEs in the array converge to a single value which is strongly affected by the composition of the PLEC layer. The $\mathrm{n} / \mathrm{p}$ ratios ranging from 0.24 to 2.19 have been observed in PLECs made with $\mathrm{Li}, \mathrm{Na}$, and $\mathrm{K}$ triflate salts as well as two different luminescent polymers. The dynamic BPE 
array thus presents a novel essay to evaluate and screen the mixed luminescent conductor for device applications.

\section{INTRODUCTION}

Bipolar electrodes (BPE) have been employed in a wide range of applications for which wireless BPEs are preferred over a conventional wired electrode. Some applications, such as the chemical locomotion of conductive objects, $1-3$ the bulk synthesis of Janus particles,4-5 and the bulk generation of electrochemiluminescence (ECL) in a liquid electrolyte necessitates the use of wireless, mobile BPEs.6 Fixed BPEs are also highly advantageous, especially in an array form 711 , or when used to generate materials with a compositional or morphological gradient.12-18

The most common BPE is any wireless metal object immersed in the electrolyte solution of an electrochemical cell containing a redox species.19-20 When the driving electrodes (DEs) of the electrochemical cells are polarized, coupled redox reactions are induced at the extremities of the BPE where the potential difference between the equi-potential BPE and the electrolyte solutions is the largest. More recently, BPEs made of other conductors have also been demonstrated. These include various types of carbon conductors, $21-25$ organic crystals26, and conducting polymers.27-28

Overwhelmingly, the BPEs are homogeneous in composition and lacking any internal structure. When polarized in a static electric field, the interior of the BPE is nearly field-free and the BPE is an equi-potential body. In this study, we demonstrate a composite, dynamic BPE. The BPE is composed of three different materials- a p-doped conjugated polymer $(\mathrm{CP})$, a silver $(\mathrm{Ag})$ microdisc, and a n-doped CP. The composite BPE is also dynamic, in that the area ratio of the $\mathrm{n}$ - 
and p-doped CP is evolving and converging to a value that is highly dependent on the active layer composition.

The dynamic BPEs are implemented in light-emitting electrochemical cells (LECs) which are solid-state electrochemical devices first developed for display and lighting applications along with the better known organic light-emitting diodes (OLEDs).29-40 The original LECs employ a luminescent CP mixed with a solid polymer electrolyte (SPE) as the active material. Such polymer LECs (PLECs) are fundamentally different from polymer-based OLEDs that do not contain any intentionally added mobile ions. The key PLEC mechanism is the redox doping reactions of the $\mathrm{CP}$ in the presence of counterions supplied by the SPE. A light-emitting $\mathrm{p}-\mathrm{n}$ junction is formed within the cell when the entire PLEC is doped. PLECs are also distinct from their liquid or gel counterparts in two important aspects: (1) the active layer of the PLEC is a mixed conductor, and (2) the light emission from a PLEC is junction electroluminescence (EL) as opposed to ECL.

Recently, BPEs have been introduced to PLECs in order to study how they affect the redox doping reactions and the device performance of PLECs. PLECs in turn offer a highly versatile, solid-state platform to study bipolar electrochemistry (BPEC)41. In a PLEC, the presence of a BPE drastically alters the doping patterns, emission profile and cell current.28, 42-43 PLECs are particularly useful in demonstrating novel BPE types such as serial-connected p-n junctions,42-43 p- and n-doped regions of the same PLEC layer, and a laser illuminated regions of the PLEC film that did not initially contain any BPE28, 44 
In this paper, we offer an in-depth analysis of the doping patterns of metal disc BPEs in planar PLECs. We show that the continued growth of the inter-connected doped areas originated from the metal BPE leads to a composite, dynamic BPE whose internal structure is a direct measure of the relative doping reaction rates.

\section{EXPERIMENTAL}

All PLECs of this study contained three materials in their active layers: a luminescent CP, a SPE polymer and a salt. Two luminescent CPs were used: poly[2-methoxy-5-(2-ethylhexyloxy)-1,4phenylenevinylene], also known as MEH-PPV, and poly[(9,9-dioctyl-2,7divinylenefluorenylene)-alt-co-\{2-methoxy5-(2-ethylhexyloxy)-1,4-phenylene $\}$, also known as ADS 108GE. MEH-PPV was synthesized by OLEDKing Optoelectronic Materials Ltd, China., and ADS108GE was provided by American Dye Source, Inc. The SPE polymer was polyethylene oxide (PEO) with a weight average molecular weight $(\mathrm{Mw})$ of $2 \mathrm{M}$ gram/mole. Lithium trifluoromethanesulfonate (LiTf), sodium trifluoromethanesulfonate (NaTf), and potassium trifluoromethanesulfonate (KTf) were the three salts used. Both PEO and salts were purchased from Aldrich and used as received. The three materials were dissolved in cyclohexanone and mixed to create casting solution of the following weight ratios: MEH-PPV (or ADS108GE):PEO:KTf=1:1.3:0.25. For PLECs made with LiTf or NaTf, the corresponding ratios are 1:1.3:0.207 and 1:1.3:0.229, respectively. These ratios ensure that all cells had the same nominal concentrations of mobile ions.

The cyclohexanone solution of above components was spin cast onto the back side of $16 \mathrm{~mm}$ by $16 \mathrm{~mm}$ glass substrates to create polymer films with the thickness ranged from $434 \mathrm{~nm}$ to 560 
$\mathrm{nm}$, as measured with a DektakXT stylus profiler. The Al DEs were thermally evaporated onto the polymer film using a shadow mask. The Ag BPE array was subsequently evaporated through a separate shadow mask onto the devices. The Al DEs and the Ag BPE array have thicknesses of $100 \mathrm{~nm}$ and $50 \mathrm{~nm}$, respectively. The above device processing steps were carried out in a MBraun integrated glove box/evaporator system filled with dry nitrogen.

The finished devices were transferred into a Janis ST-500 micro-manipulated cryogenic probe station for testing under vacuum $\left(\sim 5 \times 10_{-4}\right.$ Torr $)$. A layer of thermoconductive paste was applied between the gold-coated probe station stage and the substrate. The device was illuminated with a $365 \mathrm{~nm}$ UV ring lamp rested on the quartz window of the chamber. A LabVIEW controlled Keithley 237 source measurement unit was used to apply a voltage bias to the driving electrodes and simultaneously measure the cell current. The devices were heated and maintained at a temperature of $360 \mathrm{~K}$. The PL images were captured with a computer-controlled Nikon D300 digital SLR camera equipped with a Tamron $90 \mathrm{~mm} \mathrm{1:1} \mathrm{macro-lens.} \mathrm{The} \mathrm{captured} \mathrm{images} \mathrm{were}$ eventually processed in Matlab R2017b to extract the doping sizes.

\section{RESULTS AND DISCUSSION}

The metal BPEs are fabricated as a single horizontal array consisting of $13 \mathrm{Ag}$ discs that are 0.21 $\mathrm{mm}$ in diameter. A PLEC containing such an array is shown in Figure 1(a). The PLEC of this study has a planar or lateral (vs. sandwich) configuration with an 11-mm separation between the DEs. The extremely large planar PLECs used in this study allow for the time-lapsed photoluminescence (PL) imaging of the in situ electrochemical doping process. 

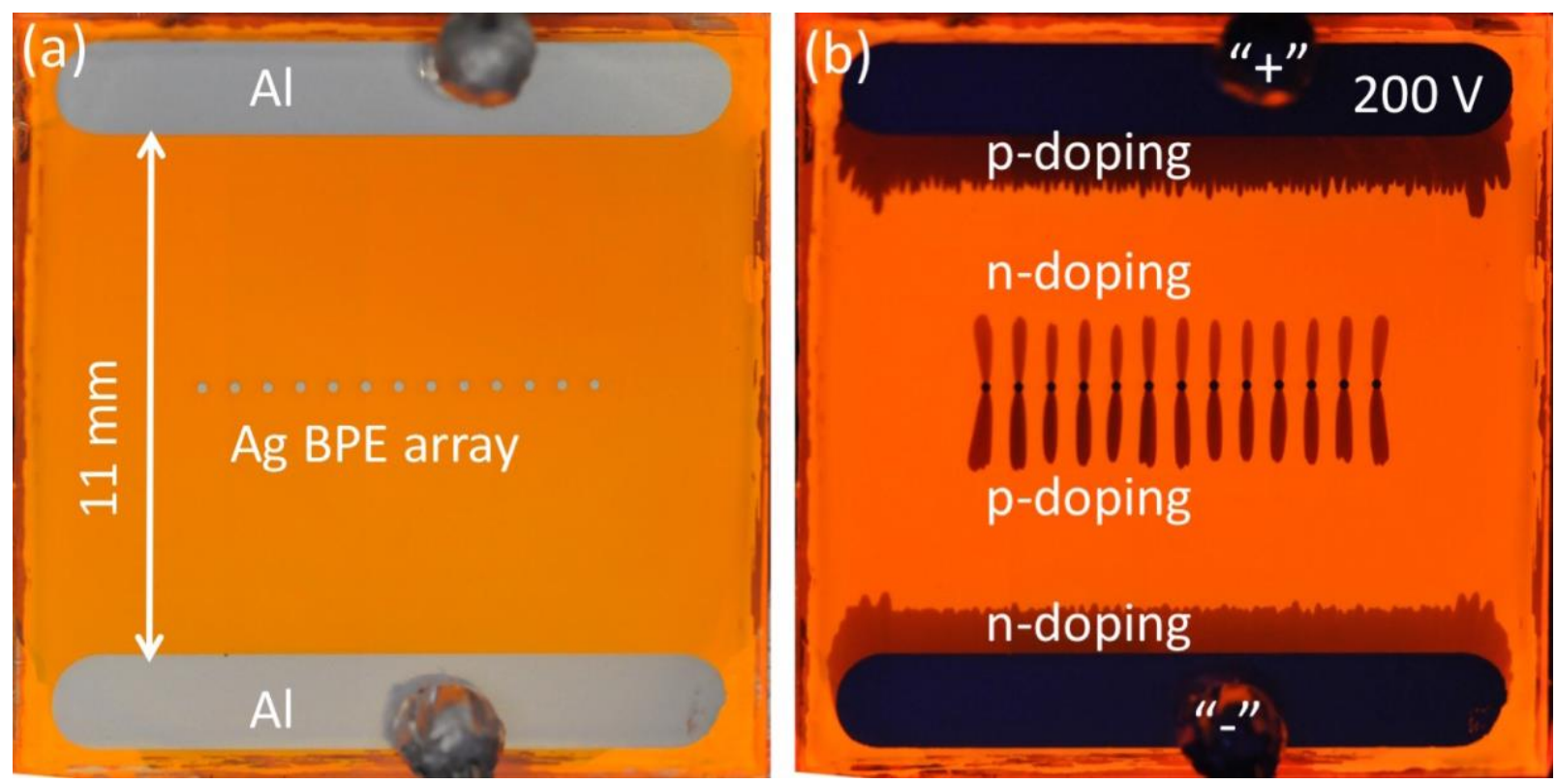

Figure 1. (a) Image of a planar PLEC under room light and UV illumination showing the active layer in orange, and the Al DEs at $11 \mathrm{~mm}$ apart. The Ag BPE array consists of identical discs $0.21 \mathrm{~mm}$ in diameter with a center-to-center separation of $0.7 \mathrm{~mm}$. Two indium balls attached to the end of gold-coated tungsten probes make electrical contact to the DEs. (b) PL image of the same PLEC $18 \mathrm{~s}$ after a voltage bias of $200 \mathrm{~V}$ was applied to the DEs with the polarity shown. The various doped regions are labelled. The device was heated to $360 \mathrm{~K}$ and tested under vacuum.

Figure 1(b) shows the PL image of a PLEC $18 \mathrm{~s}$ after a $200 \mathrm{~V}$ bias was applied to the Al DEs. Against the bright orange-red background PL of the polymer film, four distinct doping reactions are highly visible under UV illumination due to the doping reactions' strong quenching of PL. The electrochemical doping of a CP such as poly (phenylene vinylene), PPV, in the presence of counter-ions provided by the $\mathrm{KCF}_{3} \mathrm{SO}_{3}$ salt, is depicted by the following scheme: 
p-doping: $\quad(\mathrm{PV})_{n}+(\mathrm{nm}) \mathrm{CF}_{3} \mathrm{SO}_{3}^{-}-(\mathrm{nm}) \mathrm{e}^{-} \leftrightarrow\left[\left(\mathrm{PV}^{\mathrm{m}}\right)\left(\mathrm{CF}_{3} \mathrm{SO}_{3}^{-}\right)_{m}\right]_{n}$

$n-$ doping: $\quad(P V)_{n}+(n m) K^{+}+(n m) e^{-} \leftrightarrow\left[\left(P V^{m-}\right)\left(K^{+}\right)_{m}\right]_{n}$

Notably, all $13 \mathrm{Ag}$ discs display p- and n-doping in their opposite poles, confirming their function as individual BPEs. The flat geometries of the DEs generate doped regions that are roughly rectangular in shape, but with an uneven doping front. The BPE doping, on the other hand, is finger-shaped. The p-doped regions are darker than the corresponding $\mathrm{n}$-doped regions at both the DEs and the various BPEs. This color difference to do with the different electronic structures of the p- and n-doped CP, as will be shown later. The size of the fingers continues to increase with time. The quantitative analysis of the doping progression, and in particular, the $\mathrm{n} / \mathrm{p}$ area ratios provide direct evidence of a dynamic BPE whose growth and structure are highly dependent on the compositions of the PLEC active layer.

Figure 2(a) shows the time-lapsed doping pattern of a single BPE within the BPE array of a MEH-PPV PLEC containing the KTf salt. The images were taken at $3 \mathrm{~s}$ per frame after a $150 \mathrm{~V}$ bias was applied to the DEs. Strong, continued doping growth above and below the Ag BPE can be seen after some initial delay in the appearance of p-doping.

At $t=6 \mathrm{~s}$, the $\mathrm{n}$-doping has grown to nearly $0.2 \mathrm{~mm}$ in length. But the $\mathrm{p}$-doping is yet to be seen. At $\mathrm{t}=9 \mathrm{~s}$ (the second image from left), $\mathrm{p}$-doping is visible but significantly smaller in size than the n-doping above. Eventually, p-doping quickly overtakes n-doping in size.

Figure 2(b) shows the time evolution of $\mathrm{p}$ - and $\mathrm{n}$-doped areas as well as the $\mathrm{n} / \mathrm{p}$ area ratio. The areas of both BPE p- and n-doped regions increased super-linearly. This reflects the fact that as the BPE grew in size, the potential drop across the tips of the BPE also increases, causing the 
reaction rate to increase. While the size of both $n$ - and $p$-doped areas increased rapidly, the $n / p$ area ratio quickly decreased to a value of 0.61 . This is true for all BPEs in the array, despite the large spread in the size of the individual doped areas. The average $n / p$ ratio over all 13 BPEs for this device is $0.62 \pm 0.02$.

Our previous study of similar PLECs has shown that the initial doping delay is work function dependent.45 The appearance of p-doping is delayed if the BPE array is made of Ag or Al. Similarly, the appearance of n-doping is delayed if the BPE array is made of Au. But regardless of the BPE metal, the $n / p$ area ratio of all 39 BPEs in the three PLECs tested converged to a single value of $0.63 \pm 0.03$. This behavior is once again observed in Figure 2 (b) for a Ag BPE. We note that the decrease in $\mathrm{n} / \mathrm{p}$ ratio is fast but not abrupt. The intermediate $\mathrm{n} / \mathrm{p}$ ratios are still somewhat affected by the effects of the Ag disc BPE.

The remarkable convergence of $\mathrm{n} / \mathrm{p}$ area ratio signifies a transformation of the BPE, from a metal disc BPE to one that is a metal/polymer composite. When the $n / p$ area ratio converges, the BPE is dominated by the doped polymers that have grown to a much larger size than the Ag BPE. In the following we offer an in-depth analysis of the results and properties of a dynamic BPE. 

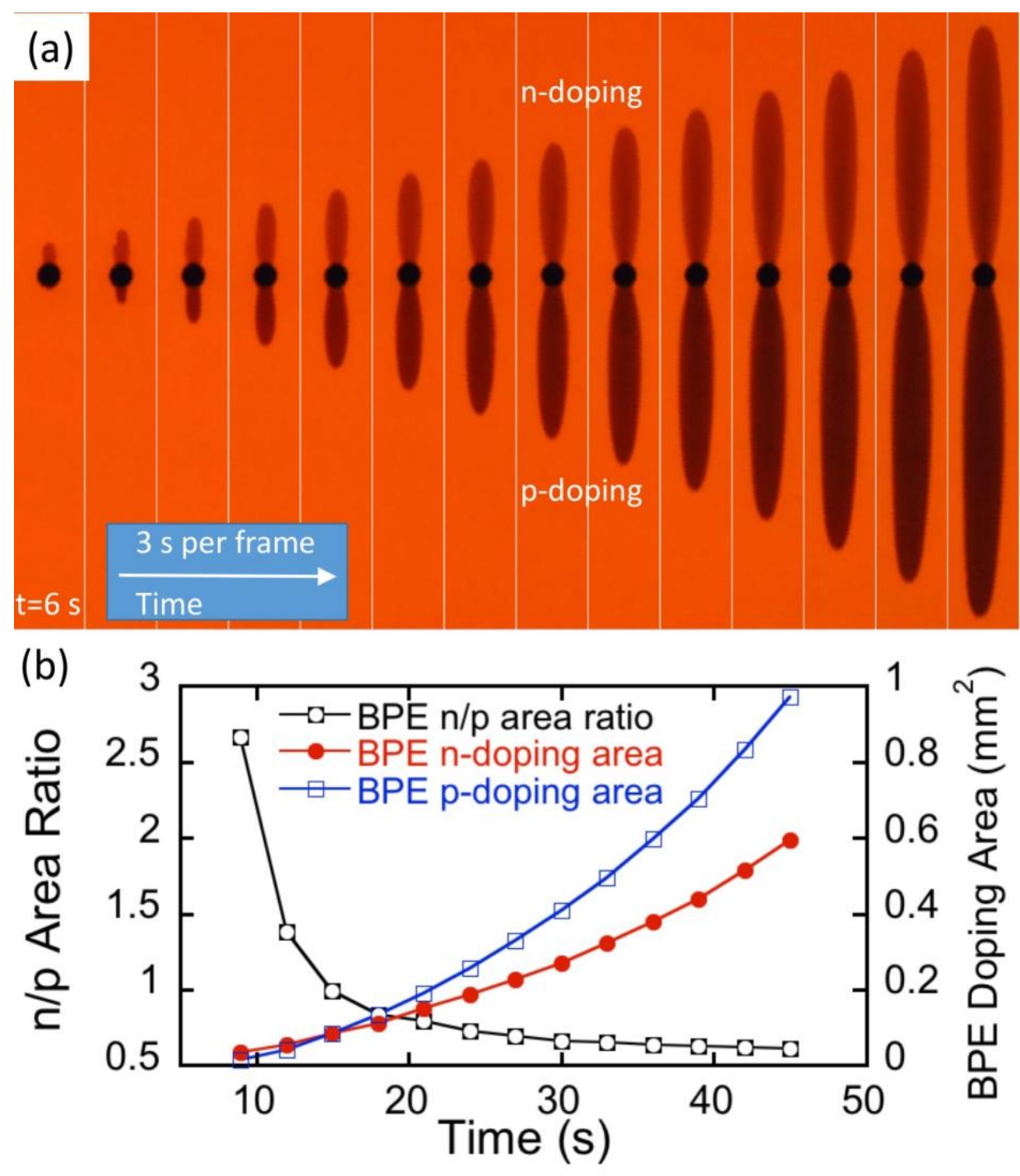

Figure 2. (a) Composite PL graph showing the doping patterns generated by a single BPE (7th from left) of the Ag BPE array. The letters " $p$ " and " $n$ " denote the BPE doping type. The PLEC contained MEH-PPV, PEO and KTf. The images were taken every $3 \mathrm{~s}$ after a $150 \mathrm{~V}$ bias was applied to the PLEC. (b) n- and p-doped areas as well as the $n / p$ area ratios calculated for the images of Figure 2(a). 
Figure 3(a) illustrates the concept of such a dynamic, composite BPE. The composite BPE consists of three conductors: a p-doped polymer, a n-doped polymer, and the Ag disc that connects the first two. The difference of the two images shows that the new BPE doping mainly occurs at the very tips of the $\mathrm{p}$ - and n-doped polymers, as expected for a BPE with this shape. Small amount of doping reactions also occurred on the sides of the doped polymers, causing the composite BPE to grow slightly thicker over time. However, no change was detected at the extremities of the original Ag BPE disc. This is consistent with the notion of a composite BPE that is now dominated by the large $\mathrm{p}$ - and n-doped polymers positioned atop and beneath the original Ag BPE.

Unlike a simple metal BPE, the composite BPE is composed of three materials of very different electronic structures. When two materials of differing Fermi levels make contact, a built-in electric field/potential develops at the interface upon reaching equilibrium. This suggests the composite BPE is not an equi-potential body as a whole. Figure 3(b) illustrates this property of the composite BPE. The p-doped polymer is represented by one with a positive bipolaron. $46-47 \mathrm{~A}$ positive bipolaron introduces two bandgap levels that are empty. Likewise, the n-doped polymer is represented by a negative bipolaron with fully occupied bandgap energy levels. The undoped polymer is free of any bandgap states. p-doping occurs when an electron from the top of the valence band (VB) of the undoped polymer enters the lower bipolaron level of the p-doped polymer. n-doping occurs when an electron from the top bipolaron level of the $\mathrm{n}$-doped polymer enters the bottom of the conduction band (CB) of the undoped polymer. 


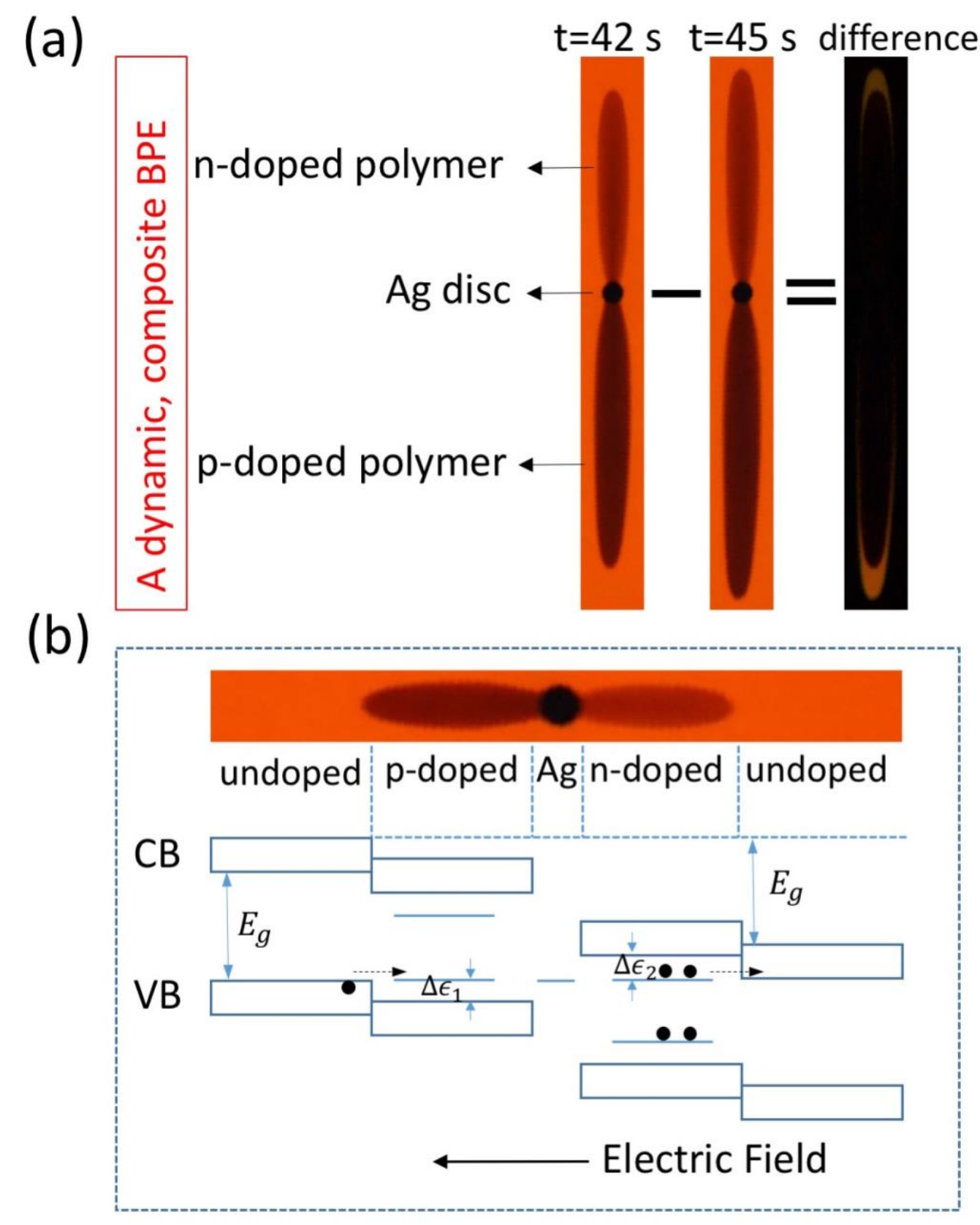

Figure 3. (a) The last two images of Figure 2(a), taken at $t=42 \mathrm{~s}$ and $t=45 \mathrm{~s}$, respectively, illustrate the concept of a dynamic, composite BPE. The difference image of the two is calculated to show new doping mainly occurs at the tips of the composite BPE. (b) The energy level diagram of the composite BPE in contact with undoped polymers to the left and right. 
For these reactions to occur, the various energy levels must be aligned, as shown in Figure 3(b). In addition, the $\mathrm{p}$ - and $\mathrm{n}$-doped polymers, as well as the composite BPE as a whole, must remain electrically neutral. This requires the upper bipolaron level of the n-doped polymer to align with the lower bipolaron level of the p-doped polymer to allow electron transport throughout the composite BPE. The simultaneous $\mathrm{p}$ - and $\mathrm{n}$-doping at the composite BPE requires a minimum energy of $\mathrm{Eg}$, as shown by the overall shift of energy levels from left to right. Note that the schematic ignored any band banding at the interfaces. For MEH-PPV, the bandgap is $2.4 \mathrm{eV}, \Delta \in 1$ and $\Delta \in 2$ are on the order of $0.7 \mathrm{eV}$. Therefore, the largest potential drop is approximately $1 \mathrm{~V}$ and it occurs between the $\mathrm{p}$ - and $\mathrm{n}$-doped polymer. The situation is similar to a reverse-biased $\mathrm{p}-\mathrm{n}$ junction, except that the $\mathrm{p}$ - and $\mathrm{n}$-doped regions are separated by a metal conductor. Note that even for a pure metal BPE, a small internal field and potential drop exist when there is current flowing through it. But the structured composite BPE has a much larger internal potential drop due to the presence of junctions.
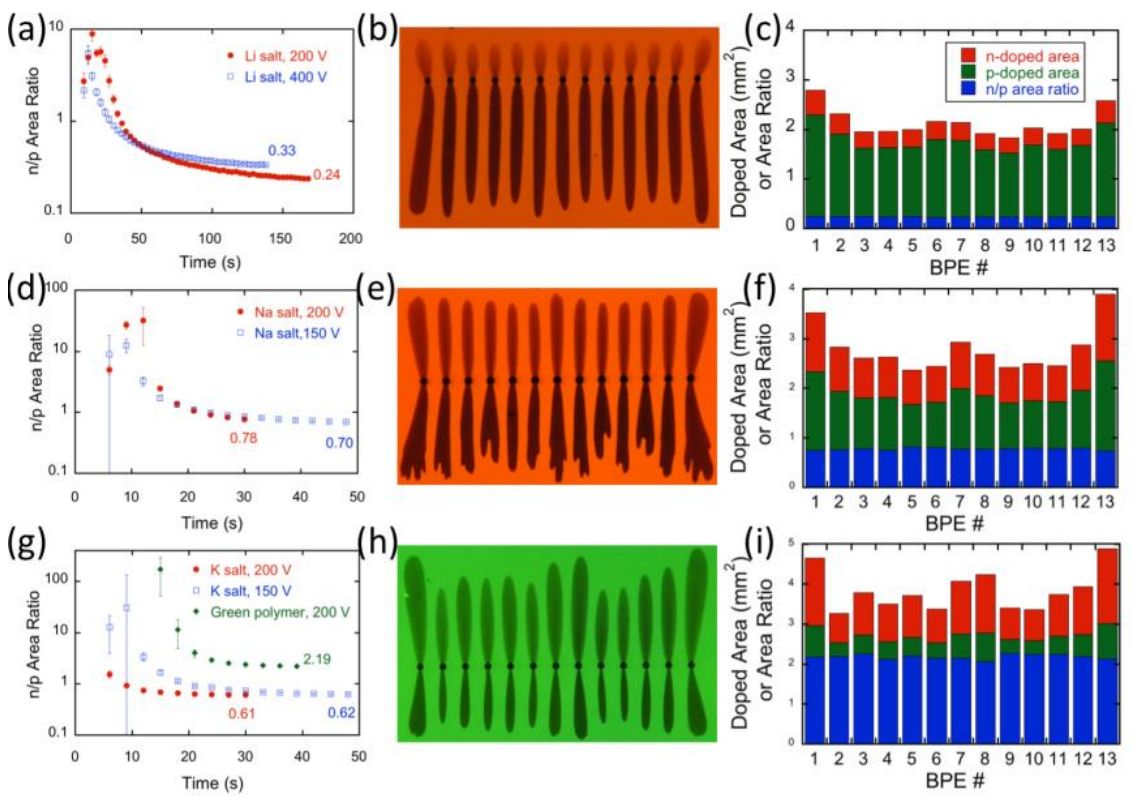
Figure 4. (a) Calculated n/p area ratios as a function of time for two MEH-PPV:PEO:LiTf PLECs tested under a voltage bias of $200 \mathrm{~V}$ and $400 \mathrm{~V}$, respectively. Each data point represents the average n/p area ratio of $13 \mathrm{BPEs}$ calculated from a single image taken at the indicated time. The error bar represents one standard deviation of the mean. (b) A cropped image showing the BPE doping that corresponds to a final $\mathrm{n} / \mathrm{p}$ ratio of 0.24 in the $200 \mathrm{~V}$ test. (c)The stacked bar diagram for Figure 4(b) showing the calculated doping areas and the area ratios for all BPEs. (d) Calculated n/p area ratios as a function of time for two MEH-PPV:PEO:NaTf PLECs tested under $200 \mathrm{~V}$ and $150 \mathrm{~V}$, respectively. (e) A cropped image showing the BPE doping that corresponds to a final $\mathrm{n} / \mathrm{p}$ ratio of 0.78 in the $200 \mathrm{~V}$ test. (f) The corresponding stacked bar diagram for Figure 4(e). (g) Calculated area ratios as a function of time for two MEHPPV:PEO:KTf PLECs tested under $200 \mathrm{~V}$ and $150 \mathrm{~V}$, respectively, and a ADS108GE:PEO:KTf PLEC tested under $200 \mathrm{~V}$. (h) A cropped image showing the BPE doping that corresponds to a final $\mathrm{n} / \mathrm{p}$ ratio of 2.19 for the ADS108GE cell in the $200 \mathrm{~V}$ test. (i) The corresponding stacked bar diagram for Figure 4(h).

The concept of dynamic BPE offers an elegant explanation of the amazing convergence of $\mathrm{n} / \mathrm{p}$ area ratio observed in all PLECs. When doping has grown to a sufficient size, it dominates the original metal discs. When this occurs the doping growth and the doping ratio will strongly depend on the composition of the PLEC active layer. Since the doping reaction requires the insertion of counter ions between the chains of the oxidized or reduced polymer, we expect the final area ratio should be strongly affected by the types of ions as well as the luminescent polymer used. 
To verify this hypothesis, we fabricated planar PLECs using three different alkali triflate salts and two luminescent polymers. MEH-PPV PLECs were fabricated using LiTf, NaTf and KTf salts. In addition, a green PLEC was fabricated using the KTf salt. All PLECs had the same Ag BPE array and were tested at $360 \mathrm{~K}$.

Figure 4 summarizes the test results of seven different LECs. The nine-panel figure is organized as follows: each row represents LECs made with different salts and/or CPs. In total, two CPs and three triflate salts are used to fabricate the LECs. The first column of graphs displays the time evolution of $\mathrm{n} / \mathrm{p}$ area ratios for all seven PLECs tested in this study. The second column of graphs displays the final images of three PLECs made with $\mathrm{Li}$, Na, and $\mathrm{K}$ salts, respectively. The last column of graphs exhibits stacked bar diagrams obtained by analyzing the images of shown in the second column. The stacked bar diagrams show calculated p-doped areas, $\mathrm{n}$-doped areas and the area ratios of all 13 BPEs of the images analyzed.

Figure 4(a) shows the time evolution of $\mathrm{n} / \mathrm{p}$ area ratios for the two MEH-PPV PLECs containing the Li salt, tested under a voltage bias of $200 \mathrm{~V}$ and $400 \mathrm{~V}$, respectively. For each device, the n/p area ratios are the averages calculated for all 13 BPEs. The final $\mathrm{n} / \mathrm{p}$ area ratios of 0.24 and 0.33 are extracted from the last images before the BPE doping and the DE doping have made contact. Figure $4(\mathrm{~b})$ displays the final image of the $200 \mathrm{~V}$ test from which a final $\mathrm{n} / \mathrm{p}$ ratio of 0.24 was calculated. Figure 4(c) is a stacked bar diagram showing the doped areas as well as the $n / p$ area ratios of all 13 BPEs in the image of Figure 4(b).

Figures 4(d)-(f) show the n/p ratios vs. time of two MEH-PPV:PEO:NaTf PLECs, the last image in the $200 \mathrm{~V}$ test, and its corresponding bar diagram, respectively. Final $\mathrm{n} / \mathrm{p}$ area ratios of 0.78 
and 0.70 are observed. Figure $4(\mathrm{~g})$ displays the calculated area ratios as a function of time for two MEH-PPV:PEO:KTf PLECs tested under $200 \mathrm{~V}$ and $150 \mathrm{~V}$, and a ADS108GE:PEO:KTf PLEC tested under $200 \mathrm{~V}$, respectively. The MEH-PPV:PEO:KTf PLECs exhibit converged n/p ratios that are nearly identical. The PLEC made with ADS108GE had the largest $\mathrm{n} / \mathrm{p}$ area ratio among all devices tested. Figure 4(h) shows the BPE doping image of the ADS108GE cell from which a final $\mathrm{n} / \mathrm{p}$ ratio of 2.19 was obtained. Figure 4(i) shows its calculated areas and ratios for all BPEs of the array.

The data presented in Figure 4 clearly demonstrate that the $n / p$ area ratio, which represents the internal composition of the dynamic BPE, is highly sensitive to both the salt and the luminescent CP of the PLECs. Although some voltage dependence has been observed in the Li salt cells, we believe the $n / p$ area ratio is largely due to the properties of the material. For the two MEHPPV:PEO:KTf PLECs tested at $360 \mathrm{~K}$, the converged $\mathrm{n} / \mathrm{p}$ area ratio of $0.61 \pm 0.02$ and $0.62 \pm 0.02$ are identical to the ratio of $0.63 \pm 0.03$ obtained in the previous study of MEH-PPV:PEO:KTf PLECs tested at $360 \mathrm{~K}$. Such highly repeatable results rule out any possibility that the divergent final $\mathrm{n} / \mathrm{p}$ ratio shown in Figure 4 is caused by the variation from device to device.

Also, all devices in this study exhibit the expected initial delay in the appearance of p-doping for Ag BPEs. Therefore, we can also rule out any cation-specific reactions between the polymer layer and the Ag BPEs. In PLECs, the electrochemical redox doping reactions require two conditions to be met: (1, the reduction and oxidation of the luminescent $\mathrm{CP}$ via electron injection or removal, and 2. the transport of counter-ions to the sites of reduction and oxidation reactions to compensate the electronic charges. The strong cation dependence of the $\mathrm{n} / \mathrm{p}$ doping ratio suggests cation transport as a main cause of the different $\mathrm{n} / \mathrm{p}$ ratios in MEH-PPV cells. This is 
supported by the data in Figure 5, which shows the cell current as a function of time for all seven PLECs of this study. The colored dots overlaid on the curves denote the moments when doping from BPEs and DEs have made initial contact to form $p-n$ junctions. When this happens, the cell current increased rapidly due to the formation of a continuous pathway for electronic current flow between the DEs. This "turn-on" behavior has also been observed in PLECs containing a vertical array of BPEs as well as those without any BPEs.43, 48

Despite the nominally identical ion concentrations, the seven PLECs displayed very different levels of current. Notably, the two Li cells had the lowest initial cell current, the slowest rise in current afterwards, and the slowest doping growth. Before any junctions were formed, the cell current is limited by the ionic conductivity of the undoped regions. Moreover, we expect the presence of BPEs and the resulting doping to have a negligible effect on the initial cell current when the voltage bias was just applied. Therefore, we can ascribe the Li cells' low current to the low conductivity of the PEO: $\mathrm{LiCF}_{3} \mathrm{SO}_{3}$ complex. This conclusion is supported by the study by Rietman et al., which showed that at $353 \mathrm{~K}$ the PEO: $\mathrm{NaCF}_{3} \mathrm{SO}_{3}$ and $\mathrm{PEO}: \mathrm{KCF}_{3} \mathrm{SO}_{3}$ complexes are about 5.6 times as conductive as the PEO:LiCF3 $\mathrm{SO}_{3}$ complex.49 This is very close to the ratio of initial cell current between the $\mathrm{Na}$ and Li cells. The low conductivity of PEO: $\mathrm{LiCF}_{3} \mathrm{SO}_{3}$ complex is likely due to the strong interaction between the $\mathrm{Li}+$ ions and the ether oxygen of PEO according to the hard-soft, acid-base (HSAB) principle.50 That is, although the ether oxygen of PEO (a hard base) can strongly solvate the $\mathrm{Li}+$ ion (a hard acid) and form a stable complex, the solvated $\mathrm{Li}+$ ions have low mobility because they are too strongly bound to the ether oxygen of PEO. By comparison, the softer, more polarizable $\mathrm{Na}+$ and $\mathrm{K}+$ ions are more mobile and contribute to a higher conductivity. 


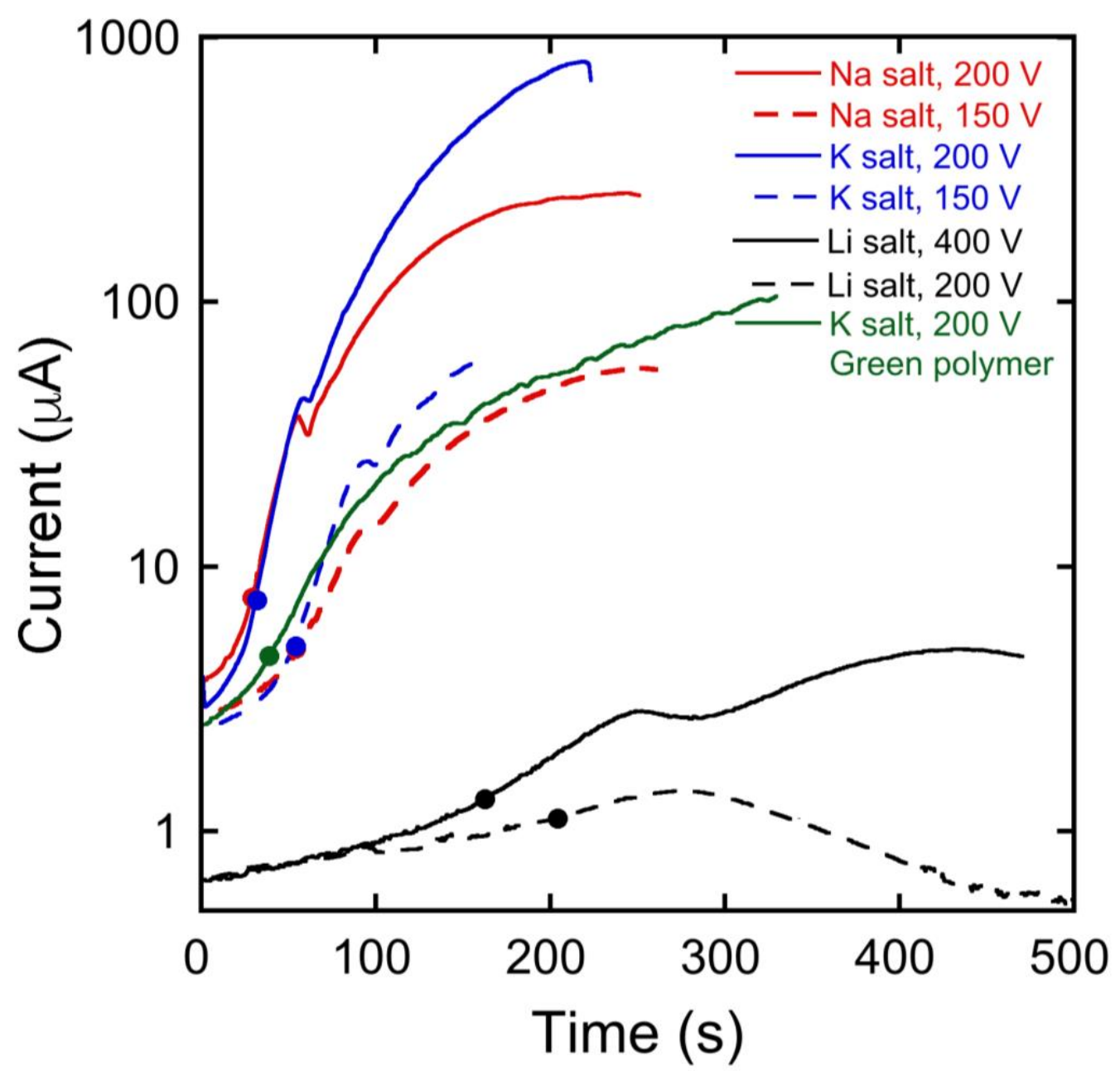

Figure 5. Time evolution of cell current for all seven PLECs tested in this study. The colored and black dots indicate the time when doping from the BPEs have made contact with doping from the DEs.

The low mobility and availability of $\mathrm{Li}+$ ions, therefore, explain the slow $\mathrm{n}$-doping growth in the Li cells. Since p- and n-doping of the dynamic BPEs are coupled, the p-doping of Li cells is also very slow, but is faster than the $n$-doping. This gives rise to the low $n / p$ ratios of 0.24 and 0.33 . If p- and n-doping of the dynamic BPE are perfectly coupled and balanced, one would not expect the $\mathrm{n} / \mathrm{p}$ ratio to be affected by the ionic mobility or conductivity. It is possible that in PLECs 
with very low ionic conductivity, the doped polymers are not sufficiently conductive to form an ideal dynamic BPE whose doping reactions only occur at the top and bottom tips of the doped regions. The conductivities of the doped regions can be inferred from the cell current after junction formation, as shown in Figure 5. When the various doped regions meet to form $p-n$ junctions in series, the cell current is no longer limited by the ionic conductivities of the polymer electrolyte, but a reflection of the electronic conductivities of the doped polymers. The low currents of the Li cells suggest an electronic conductivity several orders of magnitude lower than that of the other cells.

A very interesting result is the large $n / p$ ratio of 2.19 observed in the green polymer cell that contained $\mathrm{KCF}_{3} \mathrm{SO}_{3}$ salt. The green polymer cell displayed comparable initial current and rate of doping growth as the $\mathrm{K}$ and $\mathrm{Na}$ cells tested at $150 \mathrm{~V}$. The very large $\mathrm{n} / \mathrm{p}$ ratio cannot be explained by the effect of cations. Rather, the luminescent $\mathrm{CP}$ itself has played a major role in doping growth. Unlike MEH-PPV, the green-emitting ADS108 GE is an electron-transport emitter.51 The high mobility of electrons in ADS108GE makes it easier to be n-doped that requires the transport of electrons to the reaction sites. While MEH-PPV or a similar dialkoxy-substituted PPV are known hole transporters with a high concentration of electron traps.52-53 

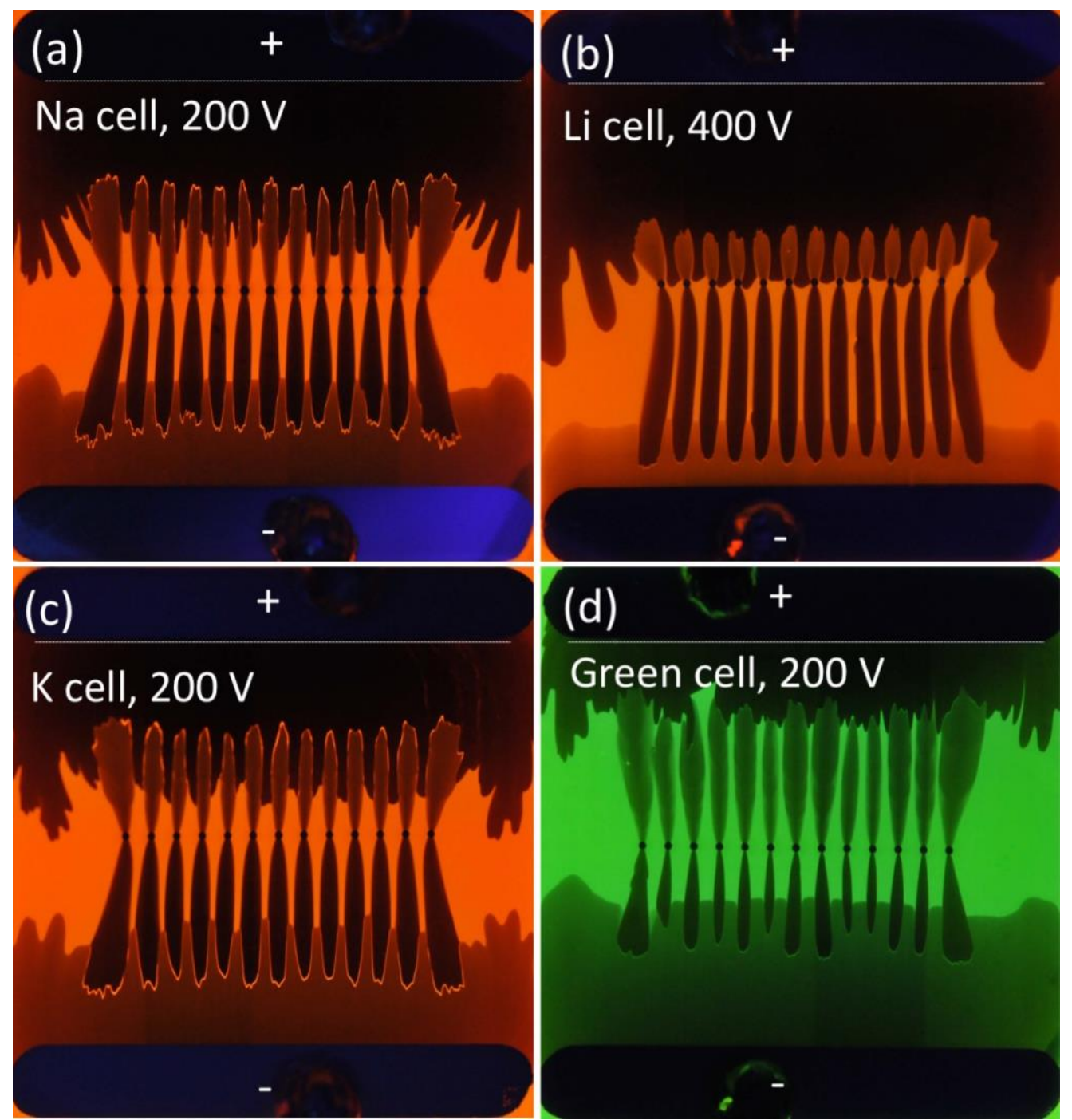

Figure 6. Full images of four PLECs showing EL from junctions formed between the dynamic BPEs and the doped regions in contact with the DEs. (a) A Na cell $52 \mathrm{~s}$ after the application of a $200 \mathrm{~V}$ bias. (b) A Li cell $231 \mathrm{~s}$ after the application of a $400 \mathrm{~V}$ bias. (c) A K cell $45 \mathrm{~s}$ after the application of a $200 \mathrm{~V}$ bias. (d) a green polymer cell $48 \mathrm{~s}$ after the application of a $200 \mathrm{~V}$ bias.

Finally, we present the EL of various PLECs in Figure 6. EL is observed at the tips of the dynamic BPEs when $\mathrm{p}-\mathrm{n}$ junctions are formed between the dynamic BPEs and the opposite doping from the DEs. EL is observed in all four cells shown. The Li cell, however, only 
displayed very faint EL at the anodic poles of the dynamic BPEs. The $\mathrm{Na}$ and $\mathrm{K}$ cells exhibit the strongest EL in all junctions formed. We conclude that strong and balanced p- and n-doping, as indicted by an $n / p$ area ratio closest to one, is probably responsible for the strongest EL in these PLECs. Balanced p- and n-doping are also sought in PLECs without any BPEs in order to avoid EL quenching by the metallic electrodes or shorts.54-55 The results of the current study are particularly relevant to bulk homojunction PLECs that contain dispersed metallic particle BPEs and $\mathrm{Li}$ salt 56 The use of a large cation, such as $\mathrm{K}_{+}$, will likely result in improved device performance when the doping reactions between the neighboring BPEs are balanced.

\section{CONCLUSION}

In this study, we have described in detail the concept and properties of dynamic BPEs. The dynamic BPEs are formed in extremely large planar PLECs and seeded by a horizontal array of evaporated Ag microdisc BPEs. A $200 \mathrm{~V}$ bias applied between the DEs of the PLECs induces strong electrochemical redox doping growth at the cathodic and anodic poles of the Ag BPEs. Over time, the p- and n-doped areas, which are highly visible under UV illumination, increase in size. Since the doped polymer is a conductor, the silver disc BPEs transform into a dynamic, composite BPE consisting of a p- and n-doped polymer conductor connected by the Ag disc. The composite BPE has a substantial internal potential drop due to the presence of junctions. The formation of a new, composite BPE is strongly supported by pattern of new doping growth, and the convergence of the $\mathrm{n} / \mathrm{p}$ area ratio for all BPEs in an array. The converged area ratio no longer reflects the properties of the BPE metal, but rather the composition of the polymer active layer. In particular, we found that the salt cations $(\mathrm{Li}+, \mathrm{Na}+$ and $\mathrm{K}+)$ as well as the luminescent $\mathrm{CP}$ have a major effect on the converged $\mathrm{n} / \mathrm{p}$ area ratio, which ranged from 0.24 to 2.19 . The dynamic 
BPE is a very useful tool to study the relative doping reaction rates of a mixed polymer conductor. This allows for convenient and reliable screening of polymer mixed conductor for device applications. The $\mathrm{n} / \mathrm{p}$ ratio, which reflects the internal composition of the dynamic BPE, should be close to 1 for maximized EL output. When the same salt is used, the dynamic BPE can be used to screen luminescent $\mathrm{CP}$ for their transport (electron vs. hole) properties.

\section{Corresponding Author}

*jungao@queensu.ca

\section{ACKNOWLEDGMENT}

This research was supported by the Natural Sciences and Engineering Research Council of Canada (Grant Number 2015-05344).

\section{REFERENCES}

1. Paxton, W. F.; Sundararajan, S.; Mallouk, T. E.; Sen, A., Chemical locomotion. Angewandte Chemie-International Edition 2006, 45 (33), 5420-5429.

2. Loget, G.; Kuhn, A., Electric field-induced chemical locomotion of conducting objects. Nat. Commun. 2011, 2, 6.

3. Sentic, M.; Loget, G.; Manojlovic, D.; Kuhn, A.; Sojic, N., Light-Emitting Electrochemical "Swimmers". Angewandte Chemie-International Edition 2012, 51 (45), 1128411288.

4. Walther, A.; Muller, A. H. E., Janus Particles: Synthesis, Self-Assembly, Physical Properties, and Applications. Chemical Reviews 2013, 113 (7), 5194-5261. 
5. Loget, G.; Roche, J.; Kuhn, A., True Bulk Synthesis of Janus Objects by Bipolar Electrochemistry. Advanced Materials 2012, 24 (37), 5111-5116.

6. Sentic, M.; Arbault, S.; Bouffier, L.; Manojlovic, D.; Kuhn, A.; Sojic, N., 3D electrogenerated chemiluminescence: from surface-confined reactions to bulk emission. Chem. Sci. 2015, 6 (8), 4433-4437.

7. Chow, K.-F.; Mavre, F.; Crooks, J. A.; Chang, B.-Y.; Crooks, R. M., A Large-Scale, Wireless Electrochemical Bipolar Electrode Microarray. Journal of the American Chemical Society 2009, 131 (24), 8364-8365.

8. Fosdick, S. E.; Berglund, S. P.; Mullins, C. B.; Crooks, R. M., Evaluating Electrocatalysts for the Hydrogen Evolution Reaction Using Bipolar Electrode Arrays: Bi- and Trimetallic Combinations of Co, Fe, Ni, Mo, and W. Acs Catalysis 2014, 4 (5), 1332-1339.

9. Khoshfetrat, S. M.; Ranjbari, M.; Shayan, M.; Mehrgardi, M. A.; Kiani, A., Wireless Electrochemiluminescence Bipolar Electrode Array for Visualized Genotyping of Single Nucleotide Polymorphism. Anal. Chem. 2015, 87 (16), 8123-8131.

10. Munktell, S.; Nyholm, L.; Bjorefors, F., Towards high throughput corrosion screening using arrays of bipolar electrodes. Journal of Electroanalytical Chemistry 2015, 747, 77-82.

11. Warakulwit, C.; Nguyen, T.; Majimel, J.; Delville, M.-H.; Lapeyre, V.; Garrigue, P.; Ravaine, V.; Limtrakul, J.; Kuhn, A., Dissymmetric carbon nanotubes by bipolar electrochemistry. Nano Letters 2008, 8 (2), 500-504.

12. Ramakrishnan, S.; Shannon, C., Display of Solid-State Materials Using Bipolar Electrochemistry. Langmuir 2010, 26 (7), 4602-4606. 
13. Ramaswamy, R.; Shannon, C., Screening the Optical Properties of Ag-Au Alloy Gradients Formed by Bipolar Electrodeposition Using Surface Enhanced Raman Spectroscopy. Langmuir 2011, 27 (3), 878-881.

14. Ulrich, C.; Andersson, O.; Nyholm, L.; Bjorefors, F., Formation of molecular gradients on bipolar electrodes. Angewandte Chemie-International Edition 2008, 47 (16), 3034-3036.

15. Ishiguro, Y.; Inagi, S.; Fuchigami, T., Gradient Doping of Conducting Polymer Films by Means of Bipolar Electrochemistry. Langmuir 2011, 27 (11), 7158-7162.

16. Xu, F.; Wang, H.; He, X. D.; Deng, N.; Li, F.; Li, B.; Xie, J. H.; Han, S. K.; He, J. B., One-step deposition of NixCu1-x alloys with both composition gradient and morphology evolution by bipolar electrochemistry. Journal of Electroanalytical Chemistry 2018, 823, 213220.

17. Lundgren, A.; Munktell, S.; Lacey, M.; Berglin, M.; Bjorefors, F., Formation of Gold Nanoparticle Size and Density Gradients via Bipolar Electrochemistry. ChemElectroChem 2016, 3 (3), 378-382.

18. Koefoed, L.; Pedersen, S. U.; Daasbjerg, K., Grafting of Aryl Diazonium, Iodonium, and Sulfonium Salts in Unusual Patterns by Exploiting the Potential Gradient in Bipolar Electrochemistry. ChemElectroChem 2016, 3 (3), 495-501.

19. Fosdick, S. E.; Knust, K. N.; Scida, K.; Crooks, R. M., Bipolar Electrochemistry. Angewandte Chemie-International Edition 2013, 52 (40), 10438-10456.

20. Loget, G.; Zigah, D.; Bouffier, L.; Sojic, N.; Kuhn, A., Bipolar Electrochemistry: From Materials Science to Motion and Beyond. Accounts of Chemical Research 2013, 46 (11), 25132523. 
21. Fattah, Z.; Garrigue, P.; Lapeyre, V.; Kuhn, A.; Bouffier, L., Controlled Orientation of Asymmetric Copper Deposits on Carbon Microobjects by Bipolar Electrochemistry. Journal of Physical Chemistry C 2012, 116 (41), 22021-22027.

22. Ongaro, M.; Gambirasi, A.; Favaro, M.; Kuhn, A.; Ugo, P., Asymmetrical modification of carbon microfibers by bipolar electrochemistry in acetonitrile. Electrochimica Acta 2014, 116, $421-428$.

23. Koefoed, L.; Pedersen, E. B.; Thyssen, L.; Vinther, J.; Kristiansen, T.; Pedersen, S. U.; Daasbjerg, K., Functionalizing Arrays of Transferred Monolayer Graphene on Insulating Surfaces by Bipolar Electrochemistry. Langmuir 2016, 32 (25), 6289-6296.

24. Ongaro, M.; Gambirasi, A.; Ugo, P., Closed Bipolar Electrochemistry for the LowPotential Asymmetrical Functionalization of Micro- and Nanowires. ChemElectroChem 2016, 3 (3), 450-456.

25. Zuccaro, L.; Kuhn, A.; Konuma, M.; Yu, H. K.; Kern, K.; Balasubramanian, K., Selective Functionalization of Graphene Peripheries by using Bipolar Electrochemistry. ChemElectroChem 2016, 3 (3), 372-377.

26. Malytska, I.; Meziere, C.; Kielar, M.; Hirsch, L.; Wantz, G.; Avarvari, N.; Kuhn, A.; Bouffier, L., Bipolar Electrochemistry with Organic Single Crystals for Wireless Synthesis of Metal-Organic Janus Objects and Asymmetric Photovoltage Generation. Journal of Physical Chemistry C 2017, 121 (23), 12921-12927.

27. Gupta, B.; Goudeau, B.; Garrigue, P.; Kuhn, A., Bipolar Conducting Polymer Crawlers Based on Triple Symmetry Breaking. Advanced Functional Materials 2018, 28 (25), 6. 
28. Hu, S. Y.; Chi, X.; Chen, S. L.; AlTal, F.; Gao, J., Visualizing the Bipolar Electrochemistry of Electrochemically Doped Luminescent Conjugated Polymers. Journal of Physical Chemistry C 2017, 121 (15), 8409-8415.

29. Pei, Q. B.; Yu, G.; Zhang, C.; Yang, Y.; Heeger, A. J., Polymer Light-Emitting Electrochemical-Cells. Science 1995, 269 (5227), 1086-1088.

30. Gao, J., Polymer light-emitting electrochemical cells-Recent advances and future trends. Current Opinion in Electrochemistry 2018, 7, 87-94.

31. Fresta, E.; Costa, R. D., Beyond traditional light-emitting electrochemical cells - a review of new device designs and emitters. Journal of Materials Chemistry C 2017, 5 (23), 5643-5675.

32. Tang, S.; Edman, L., Light-Emitting Electrochemical Cells: A Review on Recent Progress. Topics in Current Chemistry 2016, 374 (4).

33. Meier, S. B.; Tordera, D.; Pertegas, A.; Roldan-Carmona, C.; Orti, E.; Bolink, H. J., Light-emitting electrochemical cells: recent progress and future prospects. Materials Today 2014, 17 (5), 217-223.

34. Weber, M. D.; Fresta, E.; Elie, M.; Miehlich, M. E.; Renaud, J. L.; Meyer, K.; Gaillard, S.; Costa, R. D., Rationalizing Fabrication and Design Toward Highly Efficient and Stable Blue Light-Emitting Electrochemical Cells Based on NHC Copper(I) Complexes. Advanced Functional Materials 2018, 28 (17).

35. Su, H. C., Optical Techniques for Light-Emitting Electrochemical Cells. Chempluschem 2018, 83 (4), 197-210.

36. Nishikitani, Y.; Cho, T.; Uchida, S.; Nishimura, S.; Oyaizu, K.; Nishide, H., PolymerBased White-Light-Emitting Electrochemical Cells with Very High Color-Rendering Index 
Based on Blue-Green Fluorescent Polyfluorenes and Red-Phosphorescent Iridium Complexes. Chempluschem 2018, 83 (5), 463-469.

37. Nagatsu, G.; Sakanoue, T.; Tane, S.; Yonekawa, F.; Takenobu, T., An ester-substituted polyfluorene derivative for light-emitting electrochemical cells: bright blue emission and its application in a host-guest system. Materials Chemistry Frontiers 2018, 2 (5), 952-958.

38. Moore, M. D.; Bowler, M. H.; Reynolds, J. E.; Lynch, V. M.; Shen, Y. L.; Slinker, J. D.; Sessler, J. L., Ionic Organic Small Molecules as Hosts for Light-Emitting Electrochemical Cells. Acs Applied Materials \& Interfaces 2018, 10 (29), 24699-24707.

39. Jenatsch, S.; Regnat, M.; Hany, R.; Diethelm, M.; Nuesch, F.; Ruhstaller, B., TimeDependent p-i-n Structure and Emission Zone in Sandwich-Type Light-Emitting Electrochemical Cells. Acs Photonics 2018, 5 (4), 1591-1598.

40. He, L.; Wang, X. X.; Duan, L., Enhancing the Overall Performances of Blue LightEmitting Electrochemical Cells by Using an Electron-Injecting/Transporting Ionic Additive. Acs Applied Materials \& Interfaces 2018, 10 (14), 11801-11809.

41. Chen, S.; Wantz, G.; Bouffier, L.; Gao, J., Solid-State Bipolar Electrochemistry: Polymer-Based Light-Emitting Electrochemical Cells. ChemElectroChem 2016, 3 (3), 392-398.

42. Gao, J.; Chen, S.; AlTal, F.; Hu, S.; Wantz, G.; Bouffier, L., Bipolar Electrode Array Embedded in a Polymer Light-Emitting Electrochemical Cell. Acs Applied Materials \& Interfaces 2017, 9 (37), 32405-32410.

43. Hu, S. Y.; Gao, J., Wireless Electroluminescence: Polymer Light-Emitting Electrochemical Cells with Inkjet-Printed 1D and 2D Bipolar Electrode Arrays. Journal of Physical Chemistry C 2018, 122 (16), 9054-9061. 
44. AlTal, F.; Gao, J., Laser-Induced Bipolar Electrochemistry-On-Demand Formation of Bipolar Electrodes in a Solid Polymer Light-Emitting Electrochemical Cell. Journal of the American Chemical Society 2018, 140 (30), 9737-9742.

45. Hu, S. Y.; Gao, J., A dynamic bipolar electrode array for visualized screening of electrode materials in light-emitting electrochemical cells. Acs Applied Materials \& Interfaces 2018, In press.

46. Holt, A. L.; Leger, J. M.; Carter, S. A., Electrochemical and optical characterization of pand n-doped poly[2-methoxy-5-(2-ethylhexyloxy)-1,4-phenylenevinylene]. Journal of Chemical Physics 2005, 123 (4).

47. Bredas, J. L.; Street, G. B., Polarons, Bipolarons, and Solitons in Conducting Polymers. Accounts of Chemical Research 1985, 18 (10), 309-315.

48. Gao, J.; Chen, S. L.; AlTal, F.; Hu, S. Y.; Bouffier, L.; Wantz, G., Bipolar Electrode Array Embedded in a Polymer Light-Emitting Electrochemical Cell. Acs Applied Materials \& Interfaces 2017, 9 (37), 32405-32410.

49. Rietman, E. A.; Kaplan, M. L.; Cava, R. J., Alkali-Metal Ion-Poly(Ethylene Oxide) Complexes .2. Effect of Cation on Conductivity. Solid State Ionics 1987, 25 (1), 41-44.

50. Gray, F. M., Polymer Electrolytes. The Royal Society of Chemistry: New York, Weinheim (Germany), Cambridge (UK), 1997.

51. Ben Khalifa, M.; Wantz, G.; Parneix, J. P.; Hirsch, L., Multilayer fluorescent polymer light emitting diode with low voltage and high efficiency. European Physical Journal-Applied Physics 2008, 41 (1), 29-32. 
52. Blom, P. W. M.; deJong, M. J. M.; Vleggaar, J. J. M., Electron and hole transport in poly(p-phenylene vinylene) devices. Applied Physics Letters 1996, 68 (23), 3308-3310.

53. Bozano, L.; Carter, S. A.; Scott, J. C.; Malliaras, G. G.; Brock, P. J., Temperature- and field-dependent electron and hole mobilities in polymer light-emitting diodes. Applied Physics Letters 1999, 74 (8), 1132-1134.

54. $\mathrm{Hu}, \mathrm{Y}$; Gao, J., Cationic effects in polymer light-emitting electrochemical cells. Applied Physics Letters 2006, 89 (25).

55. Shin, J. H.; Robinson, N. D.; Xiao, S.; Edman, L., Polymer light-emitting electrochemical cells: Doping concentration, emission-zone position, and turn-on time. Advanced Functional Materials 2007, 17 (11), 1807-1813.

56. Tracy, C.; Gao, J., Polymer Bulk Homojunction Photonic Devices. Applied Physics Letters 2005, 87, 143502. 